\title{
INCIDÊNCIA DE COLELITÍASE PÓS CIRURGIA BARIÁTRICA: UMA COMPARAÇÃO ENTRE AS TÉCNICAS
}

Categoria: Cirúrgico.

Instituição: Centro Universitário São Camilo (CUSC).

Autores: Boza, $\mathrm{AJB}^{1}$; Silva, BC ${ }^{1}$; Rosa, $\mathrm{CR}^{1}$; Meira, GAR ${ }^{1}$; Rodrigues, $\mathrm{TF}^{1}$; Leme, $\mathrm{PLS}^{2}$.

${ }^{1}$ Discente do Curso de Medicina do Centro Universitário São Camilo.

${ }^{2}$ Orientador do artigo.

Endereço do autor principal: Rua Marechal Deodoro, 597 - São Caetano do Sul, SP.

Telefone do autor principal: (11) 97575-1719

E-mail do autor principal: annabozavest@hotmail.com 
INCIDÊNCIA DE COLELITÍASE PÓS CIRURGIA BARIÁTRICA: UMA COMPARAÇÃO ENTRE AS TÉCNICAS

Categoria: Cirúrgico.

Descritores: Bariatric Surgery, Cholelithiasis. 


\section{INCIDÊNCIA DE COLELITÍASE PÓS CIRURGIA BARIÁTRICA: UMA COMPARAÇÃO ENTRE AS TÉCNICAS}

\section{RESUMO:}

INTRODUÇÃO: No pós operatório das cirurgias bariátricas é relativamente comum os pacientes cursarem com colelitíase, a qual se desenvolve a partir da deposição de cristais de colesterol na vesícula biliar por conta da rápida perda de peso nestes pacientes. As principais técnicas utilizadas atualmente são gastrectomia em “Sleeve”, “bypass” gástrico em Y de Roux e banda gástrica, que promovem um espectro variável de perda de peso. O objetivo deste estudo é reconhecer na literatura a incidência de colelitíase nos pacientes submetidos a cirurgia bariátrica de acordo com o método aplicado.

METODOLOGIA: Revisão bibliográfica realizada a partir da base de dados PubMed, com levantamento de 89 artigos, utilizando os descritores "Bariatric Surgery" e "Cholelithiasis", publicados entre os anos de 2016 e 2021. Ao final, foram selecionados 4 artigos de acordo com a adequação ao tema.

RESULTADOS: Um dos estudos longitudinais com 3.087 pacientes submetidos à cirurgia bariátrica observou uma incidência de 10,1\% de colecistectomia após a gastrectomia em Sleeve, 9,7\% após bypass gástrico e $0,5 \%$ após banda gástrica. Por outro lado, uma metanálise e outros dois estudos de coorte retrospectivo evidenciaram uma incidência predominante de colelitíase após a cirurgia de bypass gástrico. Em todos os estudos, o procedimento de banda gástrica esteve relacionado ao menor número de colelitíase e colecistectomia. As possíveis explicações que suportam a divergência entre os artigos podem ser, tanto o número de pacientes submetidos a determinada técnica com associada colecistectomia na própria cirurgia, quanto terapias profiláticas, como o uso de ácido ursodesoxicólico (UDCA), que não foram consideradas em um dos estudos. Tais condições implicam na redução da porcentagem de pacientes que evoluem com colelitíase.

CONCLUSÃO: Apesar do crescente número de cirurgias bariátricas nos últimos anos, a literatura ainda diverge em relação a qual técnica está mais associada à colelitíase, já que todas estão relacionadas ao seu aparecimento. Ainda são necessários mais estudos para avaliar quais abordagens podem evitar ao máximo a exigência de uma colecistectomia.

DESCRITORES: Bariatric Surgery, Cholelithiasis. 


\section{INCIDÊNCIA DE COLELITÍASE PÓS CIRURGIA BARIÁTRICA: UMA COMPARAÇÃO ENTRE AS TÉCNICAS}

\section{INTRODUÇÃO}

A obesidade, definida pela Organização Mundial da Saúde (OMS) como o acúmulo anormal ou excessivo de gordura corporal em forma de tecido adiposo, e classificada por um índice de massa corporal (IMC) acima de $30 \mathrm{~kg} / \mathrm{m}^{2}$, é considerada um problema de saúde pública e tomou proporções epidêmicas, sendo combatida internacionalmente. Nas últimas décadas, sua prevalência aumentou no mundo todo. ${ }^{1}$

Juntamente com o aumento dos casos de obesidade, houve um grande aumento das cirurgias bariátricas, que constituem uma alternativa geralmente efetiva para tratar a doença e evitar suas complicações futuras. O tratamento operatório é indicado quando o IMC é maior ou igual a 40 $\mathrm{kg} / \mathrm{m}^{2}$ (obesidade grau 3) ou maior ou igual a $35 \mathrm{~kg} / \mathrm{m}^{2}$ com comorbidades associadas e um histórico de dois anos de tratamento clínico ineficaz, com obesidade estável há cinco anos. ${ }^{2}$

A cirurgia bariátrica possui diferentes técnicas, e as mais utilizadas são o bypass gástrico em Y de Roux, a gastrectomia em sleeve e a banda gástrica.

O bypass gástrico em Y de Roux, também conhecido como técnica de Fobi-Capella, é a operação mais realizada para tratar obesidade, sendo realizado um grampeamento vertical da pequena curvatura, criando uma bolsa gástrica pequena e excluindo parte do órgão. Realiza-se a reconstrução do trânsito alimentar com uma gastrojejunostomia. Faz-se a transecção do jejuno em torno de $60 \mathrm{~cm}$ após o ligamento de Treitz e uma enteroenteroanastomose, formando uma alça em Y. Como efeito principal, ocorre saciedade mais precocemente e perda do excesso do peso em cerca de 70\%, mas deve-se garantir reposição de complexo vitamínico mineral e de vitamina B12, bem como monitoramento dos níveis séricos de ferro, cálcio, vitamina D e vitamina B1. ${ }^{3}$

Recentemente, o Sleeve gástrico, também conhecido como gastrectomia vertical ou em manga, vem ganhando popularidade por ser considerado uma alternativa mais simples e com menores distúrbios nutricionais quando comparado ao bypass gástrico. ${ }^{2}$ Nesse método é feita uma incisão vertical ao longo do corpo gástrico, iniciando-se próximo ao piloro em direção ao ângulo de His, dividindo o estômago em duas partes e fechando as paredes laterais com grampeamento. Após a ressecção, o esôfago é deixado em continuidade com a curvatura menor gástrica, na forma de 
tubo, ${ }^{4}$ assim, o volume do estômago fica menor, levando à saciedade precoce e, como se retira $\mathrm{o}$ fundo gástrico, também há redução da produção de grelina, hormônio que induz o apetite. ${ }^{3}$

A banda gástrica é colocada ao redor do estômago, logo abaixo da junção gastroesofágica, através do omento menor, deixando-a acima e posterior à bolsa omental menor, que reduz as chances de erosão da banda no interior do estômago, complicação comum do procedimento. O reservatório da banda é suturado na bainha do músculo reto abdominal na porção superior do abdome, pela facilidade de acesso, para que possa ser ajustado. Nestes casos, o paciente deve agendar consultas clínicas mensalmente, pelo menos no primeiro ano, para fazer ajustes e controlar a perda de peso. ${ }^{4}$

Após a cirurgia bariátrica, sabe-se que uma consequência tardia relativamente comum que acomete os pacientes é a colelitíase. A perda de peso que acontece depois do tratamento é um fator de risco importante para a formação de cálculos na vesícula, devido ao acúmulo de sais de colesterol provenientes do fígado e uma vesícula biliar muitas vezes hipofuncionante. ${ }^{5}$

O estudo tem como objetivo reconhecer na literatura a diferença de incidência de colelitíase nos pacientes submetidos à cirurgia bariátrica, de acordo com a técnica utilizada.

\section{METODOLOGIA}

Revisão bibliográfica realizada a partir da base de dados PubMed, com levantamento de 89 artigos, utilizando os descritores "Bariatric Surgery" e "Cholelithiasis", publicados entre os anos de 2016 e 2021. Ao final, foram selecionados 4 artigos de acordo com a adequação ao tema (Figura 1).

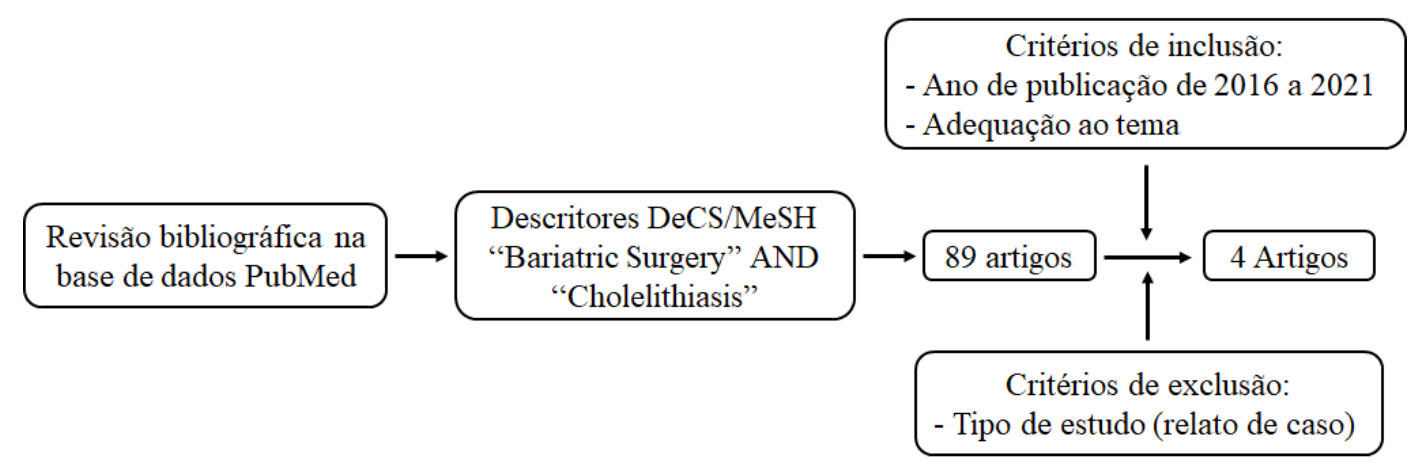

Figura 1: Fluxograma para seleção dos artigos científicos.

\section{RESULTADOS}

A tabela 1 demonstra a comparação entre as técnicas utilizadas nos procedimentos de cirurgia bariátrica, o número total de pacientes submetidos a elas e a quantidade desses que 
evoluíram com um quadro de colelitíase. Foram excluídos da análise os pacientes que já haviam passado pelo procedimento de colecistectomia, seja antes da bariátrica, ou durante esta, já como forma profilática. Em relação à tabela 2, essa descreve a relação entre a técnica utilizada na cirurgia bariátrica e a necessidade da realização de colecistectomia no período pós-cirúrgico.

Tabela 1: Incidência de colelitíase em relação às técnicas de gastrectomia em Sleeve, Bypass gástrico em Y de Roux e Banda Gástrica.

\begin{tabular}{|c|c|c|c|}
\hline Artigos & Técnicas & $\begin{array}{l}\text { Pacientes submetidos } \\
\text { à cirurgia }\end{array}$ & $\begin{array}{l}\text { Pacientes que } \\
\text { cursaram com } \\
\text { colelitíase }\end{array}$ \\
\hline Sneineh et al., $2020^{6}$ & $\begin{array}{l}\text { Gastrectomia em } \\
\text { Sleeve }\end{array}$ & 203 & $9(4,4 \%)$ \\
\hline Mishra et al., 2016 ${ }^{7}$ & & 617 & $52(8,42 \%)$ \\
\hline Sneineh et al., $2020^{6}$ & $\begin{array}{l}\text { Bypass Gástrico Y } \\
\text { de Roux }\end{array}$ & 55 & $8(14,5 \%)$ \\
\hline Mishra et al., $2016^{7}$ & & 418 & $56(13,4 \%)$ \\
\hline Sneineh et al., $2020^{6}$ & Banda Gástrica & 175 & $7(4,1 \%)$ \\
\hline Mishra et al., $2016^{7}$ & & $\begin{array}{l}\text { Não menciona a } \\
\text { técnica. }\end{array}$ & $\begin{array}{l}\text { Não menciona a } \\
\text { técnica. }\end{array}$ \\
\hline
\end{tabular}

Tabela 2: Incidência de colecistectomia pós bariátrica.

\begin{tabular}{llll}
\hline Artigo & Técnicas & $\begin{array}{l}\text { Pacientes submetidos à } \\
\text { cirurgia bariátrica sem } \\
\text { colecistectomia prévia }\end{array}$ & $\begin{array}{l}\text { Pacientes que passaram } \\
\text { por colecistectomia após } \\
\text { bariátrica }\end{array}$ \\
\hline $\begin{array}{l}\text { Tustumi } \text { et al., } \\
\mathbf{2 0 1 8}^{5}\end{array}$ & $\begin{array}{l}\text { Gastrectomia } \\
\text { em Sleeve }\end{array}$ & 1519 & $167(10,1 \%)$ \\
$\begin{array}{l}\text { Tustumi } \text { et al., } \\
\mathbf{2 0 1 8}^{5}\end{array}$ & $\begin{array}{l}\text { Bypass } \\
\text { Gástrico Y de } \\
\text { Roux }\end{array}$ & 17.224 & $1931(9,7 \%)$ \\
$\begin{array}{l}\text { Tustumi } \text { et al., } \\
\mathbf{2 0 1 8}^{5}\end{array}$ & $\begin{array}{l}\text { Banda } \\
\text { Gástrica }\end{array}$ & 14.158 & $989(6,5 \%)$ \\
\hline
\end{tabular}

\section{DISCUSSÃO}

Dois estudos de coorte retrospectivos evidenciaram uma incidência predominante de colelitíase após a cirurgia de bypass gástrico em Y de Roux, 14,5\% ${ }^{6}$ e 13,4\% $\%^{7}$, bem como a metanálise avaliada, ${ }^{5}$ que também sugeriu uma predominância de colelitíase pós-operatória após o 
bypass gástrico. O consenso entre esses três estudos é que a técnica que causa maior perda de peso e de forma mais abrupta é a que mais desenvolverá a colelitíase, por conta do aumento da concentração de colesterol na bile e a hipomotilidade da vesícula biliar.

Em contrapartida, foi observada uma incidência predominante de colecistectomia na técnica de sleeve gástrico, de 10,1\% após a gastrectomia em Sleeve, 9,7\% após bypass gástrico e 0,5\% após banda gástrica. ${ }^{8}$

Em todos os estudos a banda gástrica esteve relacionada ao menor número de colelitíase, colecistectomia ou complicações (como colecistite aguda, pancreatite aguda ou coledocolitíase), corroborando com o princípio de que a técnica que causa menor perda de peso também é a que menos cursa com formação de cálculos e complicações.

São possíveis limitações aos dados apresentados pelos estudos: número de pacientes submetidos a determinada técnica, operações em que foi associada colecistectomia durante o ato operatório, pacientes que já haviam removido a vesícula biliar antes de realizar a cirurgia bariátrica e terapias profiláticas para o aparecimento de colelitíase, como o uso de ácido ursodesoxicólico (UDCA). Embora o Bypass Gástrico tenha se mostrado a técnica em que mais foram realizadas colecistectomias pós bariátrica, o grupo amostral do mesmo evidencia que os pacientes do grupo da gastrectomia em Y de Roux foi o que mais havia realizado colecistectomia na mesma operação ou previamente à cirurgia bariátrica. ${ }^{8}$

\section{CONCLUSÃO}

Com base nos artigos analisados, foi possível determinar que o procedimento relacionado com a menor incidência de colelitíase pós-operatória é a banda gástrica. Dessa forma, conclui-se que não há consenso na literatura sobre como evitar essa complicação.

\section{DESCRITORES}

Cirurgia Bariátrica, Colelitíase.

\section{REFERÊNCIAS}

1. World Health Organization. WHO. Obesity: preventing and managing the global epidemic. Report of WHO Consultation on Obesity. Geneva, 1998. 252 p.

2. TONATTO-FILHO, Antoninho José; GALLOTTI, Felipe Melloto; CHEDID, Marcio Fernandes; GREZZANA-FILHO, Tomaz de Jesus Maria; GARCIA, Ana Maria Stapasolla 
Vargas. BARIATRIC SURGERY IN BRAZILIAN PUBLIC HEALTH SYSTEM: the good, the bad and the ugly, or a long way to go. yellow sign!. Abcd. Arquivos Brasileiros de Cirurgia Digestiva (São Paulo), [S.L.], v. 32, n. 4, 2019. FapUNIFESP (SciELO). http://dx.doi.org/10.1590/0102-672020190001e1470

3. Townsend CM, Beauchamp RD, Evers BM, Mattox KL. SABISTON. Tratado de cirurgia: A base biológica da prática cirúrgica moderna. $19^{\circ}$ edição. Rio de Janeiro: Saunders Elsevier; 2015

4. Griffin, SM; Raimes, SA; Shenfine, J. Cirurgia gastroesofágica: Um companheiro para a prática cirúrgica do especialista. $5^{\circ}$ edição. Rio de Janeiro: Saunders Elsevier; 2016

5. Tustumi F, Bernardo WM, Santo MA, Cecconello I. Cholecystectomy in Patients Submitted to Bariatric Procedure: A Systematic Review and Meta-analysis. Obes Surg. [Internet] 2018 oct [cited 2021 aug 30]; 28(10): 3312-3320. Available from: https://link.springer.com/article/10.1007\%2Fs11695-018-3443-1

6. Sneineh MA, Harel 1, Elnasasra A, Razin H, Rotmensh A, Moscovici S, Kais H, Shirin H. Increased Incidence of Symptomatic Cholelithiasis After Bariatric Roux-En-Y Gastric Bypass and Previous Bariatric Surgery: a Single Center Experience. Obes Surg. [Internet] 2020 Mar [cited 2021 aug 30]; 30(3): 846-850. Available from: https://link.springer.com/article/10.1007\%2Fs11695-019-04366-6

7. Mishra T, Lakshmi KK, Peddi KK. Prevalence of Cholelithiasis and Choledocholithiasis in Morbidly Obese South Indian Patients and the Further Development of Biliary Calculus Disease After Sleeve Gastrectomy, Gastric Bypass and Mini Gastric Bypass. Obes Surg. [Internet] 2016 oct [cited 2021 aug 30]; 26(10): 2411-7. Available from: https://link.springer.com/article/10.1007\%2Fs11695-016-2113-4

8. Altieri MS, Yang J, Nie L, Docimo S, Talamini M, Pryor AD. Incidence of Cholecystectomy following Bariatric Surgery. Surgery for Obesity and Related Diseases [Internet]. 2018 mar [cited 2021 aug 30]; 14(4): 992-996. Available from: https://www.soard.org/article/S1550-7289(18)30167-9/fulltext 